\title{
Kinematics, structure and environment of three dwarf spheroidal galaxies
}

\author{
M. E. Sharina ${ }^{1}$, I. D. Karachentsev ${ }^{1}$ and V. E. Karachentseva ${ }^{2}$ \\ ${ }^{1}$ Special Astrophysical Observatory, Russian Academy of Sciences, N.Arkhyz, KChR, 369167, \\ Russia \\ email: sme@sao.ru, ikar@sao.ru \\ ${ }^{2}$ Main Astronomical Observatory, National Academy of Sciences of Ukraine, 27 Akademika \\ Zabolotnoho St., 03680 Kyiv, Ukraine \\ email: valkarach@gmail.com
}

\begin{abstract}
We explore the environmental status of three low surface brightness dwarf spheroidal galaxies (dSphs) KKH65, KK180 and KK227 using the results of our long slit spectroscopic observations at the $6 \mathrm{~m}$ telescope of the Russian Academy of Sciences and surface photometry on the Sloan Digital Sky Survey (SDSS) images. The objects were selected by Karachentseva in 2010 as presumably isolated galaxies. The obtained surface brightness profiles demonstrate that our sample dSphs are less centrally concentrated than the objects of the same morphological type in the Virgo cluster (VC). Using the derived kinematic data we searched for possible neighbours of the dSphs within the projected distances from them $R_{\text {pro } j}<500 \mathrm{kpc}$ and with the differences in radial velocities $|\Delta V|<500 \mathrm{kms}^{-1}$. We applied the group finding algorithm by Makarov and Karachentsev to the selected sample. Our analysis shows that the dwarf galaxies of our study are not isolated. KKH65 and KK227 belong to the groups NGC3414 and NGC5371, respectively. KK180 is in the VC infall region. We conclude that it is not possible at the moment to justify the existence of isolated dSphs outside the Local Volume. The searches are complicated due to the lack of the accurate distances to the galaxies farther than $10 \mathrm{Mpc}$.
\end{abstract}

Keywords. galaxies: dwarf, galaxies: kinematics and dynamics, galaxies: fundamental parameters

\section{Introduction}

In the course of systematic spectroscopic and photometric studies of dwarf galaxies in the Local Universe we examine the properties of three objects from the list of 10 candidate isolated early-type dwarf galaxies by Karachentseva et al. (2010).

Very few isolated dSphs were found up to date (Karachentseva et al. 1999, Karachentsev et al. 2001, Makarov et al. 2012). It has been proved observationally that most dSphs are located within $\sim 2$ virial radii from a massive neighbour (Karachentsev et al. 2005). The evolution of dwarf galaxies is influenced by the environmental factors, and by internal starbursts (e.g. Grebel 2005, Kormendy \& Bender 2012 and references therein).

The origin of isolated dSphs is still not fully understood. Ricotti \& Gnedin (2005) suggested that these objects may originate in low-mass halos $\left(<2 \cdot 10^{8} M_{\odot}\right)$ before the reionization epoch.

\section{Results and conclusions}

Our spectroscopic observations were carried out with the SCORPIO spectrograph (Afanasiev \& Moiseev 2005) equipped with the CCD detector EEV $42-40$ and the grism VPHG1200B. The reduction of the photometric and spectroscopic data was performed 
using the MIDAS (Banse et al. 1983) and IRAF (Tody 1993) software systems. The radial velocities were derived using the ULYSS program (Koleva et al. 2008, 2009).

Photometry on SDSS images and fitting of the surface brightness profiles showed that $g-r, r-i$ colours of the three galaxies are typical for dSphs (Sharina et al. 2008, 2013 and references therein). The Sersic indices are in the range $0.9 \div 1.1$. Our sample objects are less centrally concentrated than dSphs in the VC (Kormendy et al. 2009).

We used the derived radial velocities and integrated magnitudes to identify possible neighbours of KKH65, KK180 and KK227. First, we searched for galaxies in the SDSS and LEDA databases within the projected radii $R_{\text {proj }}<500 \mathrm{kpc}$ around dSphs and with the differences in radial velocities $|\Delta V|<500 \mathrm{kms}^{-1}$. Then we applied the group finding algorithm by Makarov \& Karachentsev (2011) to the selected sample. We considered the studied galaxies to be located at their Hubble distances.

It appears that KKH65 $\left(V_{L G} \sim 1300 \mathrm{kms}^{-1}, V_{t}=16.9\right)$ is one of the members of the NGC3414 group. The projected separation of KKH65 from the massive lenticular galaxy is $\sim 130 \mathrm{kpc}$. KK180 $\left(V_{L G} \sim 609 \mathrm{kms}^{-1}, V_{t}=16.1\right)$ has no close bright massive neighbours within $R_{\text {proj }}<500 \mathrm{kpc}$ and with the differences in radial velocities $|\Delta V|<500 \mathrm{kms}^{-1}$. However, the VC is so massive that KK180 is surely under its gravitational influence. KK227 $\left(V_{L G} \sim 1900 \mathrm{kms}^{-1}, V_{t}=17.1\right)$ belongs to the group of $\sim 50$ galaxies around NGC5371.

The exact environmental status of the three dSphs will be established when the accurate distances are known for them and for their neighbouring galaxies.

Acknowledgements. This work was performed under support of the Russian Science Foundation grant N 14-12-00965. IDK thanks a grant RFBR 13-02-90407 Ukr f a. VEK acknowledges a Ukrainian-Russian grant F 53.2/015. We are grateful to Dr. S.N. Dodonov for the technical supervision of our observations. We acknowledge the usage of the HyperLeda (http://leda.univ-lyon1.fr) and the SDSS (http://www.sdss.org) databases.

\section{References}

Afanasiev, V. L. \& Moiseev, A. V. 2005, Astron. Lett., 31, 194

Banse, K., Crane, P., Grosbol, P., Middleburg, F., Ounnas, C., Ponz, D., \& Waldthausen, H. 1983, The Messenger, 31, 26

Grebel, E. K. 2005 in Jerjen H., Binggeli B. (eds.), Near-fields cosmology with dwarf elliptical galaxies, Proc. IAU Colloq. 198 (Cambridge Univ. Press), p. 1

Karachentsev, I. D., Karachentseva, V. E., \& Sharina, M. E. 2005, in Jerjen H., Binggeli B. (eds.), Near-fields cosmology with dwarf elliptical galaxies, Proc. IAU Colloq. 198 (Cambridge Univ. Press), p. 295

Karachentsev, I. D., et al. 2001, A\& A, 379, 407

Karachentseva, V. E., Karachentsev, I. D., \& Sharina, M. E. 2010, Astrophysics, 53, 462

Karachentseva, V. E., Karachentsev, I. D., \& Richter, G. M. 1999, A\&\&AS, 135, 221

Koleva, M., Prugniel, P., Bouchard, A., \& Wu, Y. 2009, A\& A, 501, 1269

Koleva, M., Prugniel, P., Ocvirk, P., Le Borgne, D., \& Soubiran, C. 2008, MNRAS, 385, 1998

Kormendy, J. \& Bender, R. 2012, ApJS, 198, 2

Kormendy, J., Fisher, D. B., Cornell, M. E., \& Bender, R. 2009, ApJS, 182, 216

Makarov, D., Makarova, L., Sharina, M., Uklein, R., Tikhonov, A., Guhathakurta, P., Kirby, E., \& Terekhova, N. 2012, MNRAS, 425, 709

Makarov, D. I. \& Karachentsev, I. D. 2011, MNRAS, 412, 2498

Ricotti, M. \& Gnedin, N. Y. 2005, ApJ, 629, 259

Tody, D. 1993, ASP-CS, 52, 173

Sharina, M. E., Karachentseva, V. E., \& Makarov, D. I. 2013, Advancing the Physics of Cosmic Distances, Proc. IAU Symp.289 (Cambridge Univ. Press), p. 236

Sharina, et al. 2008, MNRAS, 384, 1544 